\title{
Reflets
}

Revue ontaroise d'intervention sociale et communautaire

\section{La transformation du travail et les jeunes : quelle place pour le service social?}

\section{Lilian Negura et Nérée St-Amand}

Volume 14, numéro 1, 2008

Travail, jeunesse et intervention

URI : https://id.erudit.org/iderudit/018852ar

Aller au sommaire du numéro

Éditeur(s)

Reflets : Revue d'intervention sociale et communautaire

ISSN

1203-4576 (imprimé)

1712-8498 (numérique)

Découvrir la revue

Citer ce document

Negura, L. \& St-Amand, N. (2008). La transformation du travail et les jeunes :

quelle place pour le service social? Reflets, 14(1), 10-24. 


\section{La transformation du travail et les jeunes : quelle place pour le service social?}

\section{Lilian Negura et Nérée St-Amand}

Depuis quelques années, le travail constitue le lieu d'une réflexion importante et diversifiée dans plusieurs champs d'études: l'économie, les sciences de l'éducation, la sociologie, la psychologie, le droit, etc. Cet intérêt marqué s'explique par l'ampleur des transformations sociales qui accompagnent la réorganisation de l'économie locale et mondiale et par l'importance grandissante du travail rémunéré dans nos sociétés contemporaines. La mondialisation, les nouvelles technologies, la flexibilité, la précarisation de l'emploi, la concurrence, le néolibéralisme, la délocalisation, l'économie du savoir, l'individualisation ou les nouvelles gestions sont autant de manifestations de la nouvelle donne du monde de travail qui se sont converties en des sujets à la mode, en sciences sociales en particulier. L'émergence de cette dynamique réflexive ouvre la voie à un examen de la place du service social dans ce débat.

Pendant les "trente glorieuses ", ${ }^{1}$ l'entreprise a été le lieu privilégié du lien social et de l'insertion sociale, grâce à un système productif qui a conjugué la production de masse et certaines politiques de protection sociale des salariés (Dubar, 2000). Cette période est caractérisée par le plein emploi, l'importance de la formation initiale, l'insertion professionnelle rapide, des carrières ascendantes au sein de la même entreprise, le temps plein, un horaire de travail stable et prévisible, un emploi presque garanti à vie, protégé par des syndicats forts et un État social très actif. Au cours de ces années de prospérité, la classe moyenne s'est 
élargie pour se transformer en un facteur important de stabilité dans les sociétés occidentales (Chauvel, 2006). L'intégration professionnelle était à l'époque synonyme de l'intégration sociale (Castel, 2000) et, en contrepartie, la pauvreté était étroitement liée au chômage. C'est au cours de cette période que des approches socio-politiques, comme les approches structurelles, féministes, communautaires ou d'action sociale, se taillent une place dans le champ du service social, jusqu'alors principalement centré sur des interventions de case-work selon des approches cliniques ou directes (Lecomte, 2000).

Depuis les années 1980, la mondialisation et la montée en importance des nouvelles technologies d'information et de communication provoquent rapidement un bouleversement du fonctionnement des entreprises (Negura, 2006). Ce processus culmine dans les années 1990 par l'instauration d'un système productif postfordiste caractérisé par l'introduction de nouveaux types de gestion fondés sur le principe de la flexibilité (Dubar, 2000). La majorité des entreprises ne gardent qu'un noyau dur d'emplois stables pour externaliser les fonctions qui ne sont pas jugées indispensables à la production. Ce changement structurel encourage la sous-traitance, fragilise le statut d'emploi et désengage les entreprises de leurs responsabilités sociales envers les gens et les communautés locales (Rose, 2000). De plus, l'effritement de l'emploi et l'individualisation de la production affaiblissent beaucoup la marge de manœuvre des syndicats qui perdent peu à peu leur influence dans les entreprises (Pernot, 2005). Le transfert des entreprises vers des pays à faible revenu augmente le chômage de certaines catégories de travailleurs et multiplie les catégories sociales exigeant un régime de protection sociale. Comme l'État social a été créé dans un contexte de prédominance de l'emploi stable, les nouvelles conditions diminuent aussi la capacité du système social de remplir adéquatement ces fonctions (Rosanvallon, 1995).

En conséquence, de nombreux changements surviennent dans le fonctionnement de l'entreprise. Les nouvelles technologies de communication, comme l'intranet, permettent la réorganisation de la communication dans les organisations (Duval et Jacot, 2000). 
Une conséquence est une redistribution de la responsabilité décisionnelle du centre managérial aux salariés. Ce fait est ressenti par les travailleuses et travailleurs comme une pression, accentuée par la recherche de la qualité totale et l'adaptation constante de la production aux besoins fluctuants du marché qui devient le seul référant. En effet, le travailleur est mis dans la situation d'être parfait, de n'admettre aucune défaillance et d'exécuter ses tâches selon le principe just-in-time pour réussir ainsi une interdépendance en temps réel des phases de production et de commercialisation des produits et des services (Rinehart, 2001). Le travail devient individualisé, moins standardisé, plus créatif, flexible, mais aussi plus intense, imprévisible, insécurisé, centré sur le court terme, instable, exigeant et précaire.

Cette période s'accompagne d'un désengagement de l'État et de la décentralisation du social. Même si le Canada enregistre d'importants surplus budgétaires, les gouvernements refusent de les réinvestir dans le social (logement, assistance, protection des travailleurs, des femmes, des minorités, etc.). Cette réalité amène de nouvelles préoccupations dans le champ du service social.L'émergence des approches postmodernes avec leur intérêt pour la différence et la diversité relativise les problèmes sociaux et insiste sur l'éclectisme et l'écologisme culturel (Lecomte, 2000). Cependant, elles n'ont pas réussi à prendre en charge ces changements de structure, ce qui fait en sorte que les travailleuses et les travailleurs sociaux ressentent un sentiment d'impuissance face à l'ampleur des problèmes. Ce fait provoque paradoxalement le retour des approches centrées sur l'individu, les petits groupes et les familles (Lecomte, 2000).

On remarque aussi que le travail n'a pas nécessairement été au cœur des préoccupations de la profession du service social. Cependant, il est évident que les nouvelles réalités du travail ont des conséquences sur des classes entières de citoyens. L'organisation du travail, fondée sur la flexibilité, a des effets dramatiques sur le bien-être psychologique de beaucoup de personnes (Dejours, 2003; Molinier, 2007). Les troubles psychologiques des salariés des nouvelles entreprises remplacent les accidents physiques au travail. Les notions de burn-out ou du stress professionnel font 
désormais partie intégrante du langage quotidien des salariés. Les personnes moins scolarisées sont éliminées systématiquement du marché du travail pour accorder plus de place à l'implantation d'un capitalisme cognitif (Moulier-Boutang, 2007). Ces personnes vulnérables rejoignent le groupe des nouveaux chômeurs, victimes de la délocalisation des entreprises vers les pays où la main-d'œuvre est à meilleur marché. Cette « sélection naturelle » des travailleurs a déjà des conséquences significatives sur la cohésion sociale des communautés, effets pervers d'une économie dissociée du social (Guibert et Mergier, 2006).

Avec l'évolution du travail, la pauvreté prend aussi un autre visage. Il ne s'agit plus d'une pauvreté limitée aux personnes exclues ou en marge de la société. Elle s'est élargie aux travailleurs, phénomène qui a été qualifié de nouvelle pauvreté (Paugam, 2002). De plus, l'emploi en soi est devenu une réalité aux contours fragiles et instables. Le travail atypique, qu'il soit à temps partiel, à durée déterminée, sur appel, à distance ou indépendant devient de plus en plus typique pour l'actuel système postfordiste de production (Fournier, Bourassa et Béji, 2003). Ces emplois sont caractérisés par leur statut incertain, provisoire et qualifié de flexible.

Ces changements structurels sont accompagnés par des évolutions rapides des valeurs et des représentations. Les nouvelles générations des jeunes véhiculent une vision plus hédoniste du travail en valorisant l'autonomie, la créativité, l'autoaccomplissement, le plaisir, les liens sociaux et la liberté (Negura, 2006). Il s'agit de l'aboutissement d'une évolution graduelle de longue date qui prend ses racines dans le mouvement de désaffection à l'égard du travail des années 1960. Une évolution qui, paradoxalement, pourrait converger avec les tendances actuelles d'individualisation et d'intellectualisation de l'économie. Pour le moment cependant, les jeunes, à côté d'autres groupes comme les femmes ou les minorités ethniques, se retrouvent, quand ils ont la chance de s'y retrouver, dans les emplois les plus précaires, qui correspondent très difficilement à ces valeurs qu'ils privilégient.

En 2003, le taux de chômage de la population active au Canada était de 7,6\%, alors que le taux de chômage des jeunes de 15 à 
24 ans gravitait autour de $14 \%$ (Statistique Canada, 2004). Si le chômage des adultes se caractérise généralement par une perte d'emploi, on observe plutôt chez les jeunes un prolongement de la période de transition entre les études et le travail (Grenier, 1998). Il s'agit de la chronicisation d'un profil regroupant certains éléments : succession des emplois de courte durée, travail à temps partiel à défaut d'emploi à temps plein, travail à forfait, programmes d'emplois subventionnés en alternance avec des périodes de chômage, travail au noir, retrait de la population active (Tremblay, 2003). Il ne s'agit pas ici du profil d'emploi étudiant qui est par définition temporaire et à temps partiel, mais de la situation des jeunes qui désirent se stabiliser sur le marché du travail. En fait, seulement $42 \%$ de jeunes n'étant plus aux études et âgés de 15 à 24 ans se retrouvaient en 2003 dans un emploi à temps plein (Statistique Canada, 2004). De plus, les emplois occupés par les jeunes sont fréquemment des emplois à faible qualification, ce qui entraîne des possibilités réduites de planification d'une carrière à long terme.

De telles tendances affectent surtout les jeunes les moins scolarisés ou qui ont abandonné leurs études. Si pour les étudiantes et étudiants le défi se pose plutôt d'assurer l'équilibre études/ travail, les jeunes qui n'ont pas terminé leurs études secondaires ne possèdent souvent pas les compétences minimales requises ou l'expérience de travail exigée par les employeurs. Ils présentent alors le taux de chômage le plus élevé2. La précarité des emplois des jeunes et leur faible taux de syndicalisation expliquent aussi l'absence de protection sociale et d'avantages sociaux chez cette population (Raymond, 2008). La situation particulière des jeunes en général se complexifie davantage dans le cas de ceux et celles vivant avec un handicap, des jeunes Autochtones, des jeunes immigrants ou des jeunes toxicomanes. Devant concurrencer avec les autres, ces derniers sont désavantagés de surcroit par des dynamiques sociales de stigmatisation et de discrimination sur le marché du travail.

Face à la complexité de cette situation, nous avons trouvé important de dédier ce numéro de Reflets à une réflexion sur le rôle que peut jouer le service social vis-à-vis la problématique 
des jeunes au travail dans le nouveau contexte des changements structurels de l'économie. C'est dans ce contexte que le présent numéro propose de répondre à certains objectifs, en particulier:

1. Attirer l'attention sur certaines populations plus vulnérables face à la fragilisation de monde du travail;

2. Alimenter nos réflexions sur les retombées de pratiques en évolution rapide;

3. Réfléchir sur les impacts de telles transformations, tant en Ontario français que dans d'autres contextes;

4. Proposer un engagement de notre profession face à ces situations changeantes.

Ce numéro se veut donc un espace de réflexion engagée par rapport aux jeunes en situation de précarité face à un monde du travail en évolution rapide et souvent exponentielle. Que fait le service social vis-à-vis de telles réalités complexes, nécessitant à la fois une analyse politique, économique et idéologique qui orienterait nos interventions? Quelles influences de telles tendances ont-elles, tant sur notre pratique, sur nos recherches que sur nos enseignements? De plus, la dimension minoritaire que privilégie la revue ajoute une variable supplémentaire à une problématique déjà fort complexe.

Ce numéro ne prétend certes pas pouvoir vider toutes ces questions ni répondre à toutes les préoccupations qu'il soulève. Par contre, l'entrevue, les articles de fond et la rubrique Des pratiques à notre image proposent des solutions possibles et des lieux de réflexion visant tant à alimenter le débat autour d'une société à caractère humain que d'une économie responsable et d'un service social engagé.

\section{L'entrevue}

L'entrevue avec Daniel Mercure propose une réflexion sur l'évolution actuelle du monde du travail en jetant les bases d'une compréhension du rôle du service social dans ce nouveau contexte. D'entrée de jeu, Daniel Mercure explique son concept 
d'impartition flexible et montre comment ce phénomène est à l'origine d'une plus grande précarité au travail. Le nouveau volet de cette précarité est qu'elle touche désormais des catégories de personnes qui étaient jusqu'alors relativement à l'abri - les travailleuses et les travailleurs. Comme conséquence, on observe une extension de la vulnérabilité, qui devient un état difficile à définir, car situé entre l'intégration et l'exclusion. La précarité n'est pas la seule conséquence négative de l'évolution du monde du travail. L'exploitation de la subjectivité et la polyvalence, résultats de la flexibilité fonctionnelle des entreprises, ont des effets dévastateurs sur la santé mentale des personnes.

Daniel Mercure poursuit en proposant une réflexion sur la centralité du travail. Pendant que la centralité objective du travail prend plus de place, la centralité subjective diminue. En fait, il s'agit d'un paradoxe :les gens travaillent de plus en plus, mais, en même temps, ils ne désirent pas être envahis par le travail. L'auteur avance son explication face aux difficultés de la nouvelle génération de travailleurs d'accepter la loyauté à l'endroit des entreprises. En fait, il s'agit d'une réaction aux pratiques contradictoires des entreprises : elles ont développé dans les années 1980 le sentiment d'appartenance pour démontrer ensuite dans les années 1990 leur manque de fidélité.

Pour Daniel Mercure la spécificité des «nouveaux vulnérables» est surtout leur isolement, le fait qu'ils ne sont plus intégrés dans des communautés fortes, comme les syndicats ou les groupes professionnels. Les solutions proposées jusqu'ici pour ce nouveau type de vulnérabilité sont plus individuelles qu'institutionnelles : on privilégie la responsabilisation individuelle. Selon lui, "les travailleurs sociaux ne peuvent plus faire l'économie d'une bonne compréhension du marché du travail ", leur défi étant "d'arrimer leur compréhension des dynamiques socioculturelles et psychologiques à celle du marché du travail et penser de manière novatrice leur rôle et leur pratique dans un tel contexte ». Ses prédictions pour l'avenir du travail sont à la fois pessimistes et optimistes : le travail sera plus présent dans nos vies, tandis que les entreprises apprendront à développer de nouvelles mesures de protection sociale plus adaptées à la nouvelle réalité du travail. 


\section{Le dossier}

Dans leur article Pauline Morissette et ses collègues s'interrogent sur le phénomène de la précarisation des trajectoires socioprofessionnelles de jeunes toxicomanes de 18 à 30 ans. Les changements économiques qui ont créé les conditions pour la généralisation d'une organisation du travail génératrice de précarité touchent essentiellement les catégories vulnérables des travailleurs, dont les jeunes toxicomanes. Comme le processus d'insertion socioprofessionnelle est un élément inhérent à la sortie réussie de la toxicomanie, les auteures constatent l'importance d'analyser ces dynamiques. L'article illustre les trajectoires socioprofessionnelles des jeunes toxicomanes pour rendre compte du rôle dans leur vie de la réapparition de cette double précarisation à la fois professionnelle et sociale. Le modèle d'analyse inductive des récits de vie d'une trentaine de jeunes consommateurs de substances psychoactives privilégie quatre pôles : les déterminants contextuels et individuels, les stratégies de défense et d'action. Ces pôles ont permis aux auteures de mettre en relief des interactions à l'origine de quatre trajectoires et de décrire la manière dont la dynamique de précarisation se met en place. Ces cas de figure illustrent comment l'histoire personnelle et familiale des jeunes toxicomanes s'imbrique sur un fond structurel de précarisation professionnelle afin de produire les conditions favorables à la consommation. Cette recherche démontre ainsi l'importance pour la compréhension de la toxicomanie des jeunes travailleurs de conjuguer l'analyse des facteurs individuels de ce phénomène avec l'étude du contexte structurel du monde du travail. Cette recherche est aussi une invitation à intervenir "autrement", d'une manière compréhensive, en prenant en compte le contexte structurel sans négliger la dimension diachronique de l'histoire personnelle de ces jeunes.

Dans une analyse de la problématique de la rupture de la formation professionnelle duale en Suisse, et privilégiant une lecture selon l'approche de la psychodynamique du travail, Nadia Lamamra et Jonas Masdonati cherchent à définir ce que 
pourraient être des stratégies de défense mises en place par les apprenantes et les apprenants en situation de souffrance. Les auteurs montrent la double nature de la formation duale, étape importante de socialisation professionnelle et de constitution d'une identité professionnelle, mais aussi lieu de passage accéléré à la vie professionnelle adulte, avec tous les risques que cela comporte : la compétition, les contraintes de productivité et de rendement, et parfois la rupture. L'article met à profit les concepts théoriques de la psychodynamique du travail pour analyser le discours de dix-huit apprenantes et apprenants qui ont arrêté leur formation professionnelle duale durant leur première année d'apprentissage. Les auteurs se sont proposé de repérer l'évocation subjective de la souffrance et de la peur amenées par les conditions précaires de travail, les relations tendues, la situation de transition et d'explorer les stratégies utilisées par ces jeunes pour les affronter. Les stratégies dégagées de la littérature sur la souffrance au travail n'ont pas permis d'identifier l'arsenal des moyens mis en place par les apprenantes et apprenants. Grâce à une démarche inductive, les auteurs ont découvert de nouvelles stratégies spécifiques de cette population, qui n'avaient pas été répertoriées auparavant par les chercheurs. Ces stratégies, combinées avec les autres, ont permis de balayer des profils distincts de ces jeunes. Sur la base de cette analyse, les auteurs nous convient à reconnaitre certains faits qui nous aident à mieux comprendre la spécificité de la population étudiée : des adolescents conscients de l'importance de leur formation (cela explique l'utilisation de la stratégie de l'endurance), des adolescents à leur premier contact avec le monde du travail (cela explique la sous-utilisation des stratégies comme la concurrence, le clivage, l'individualisme, la collaboration ou le cynisme viril), des adolescents à un âge qui leur permet de solliciter plus aisément des appuis institutionnels ou personnels (cela explique l'utilisation de nouvelles stratégies comme l'action et le partage) et des adolescents ayant un statut encore ambigu qui se situe entre l'indépendance adulte et la dépendance infantile (cela explique l'utilisation de plusieurs stratégies souvent contradictoires). La temporalité constitue ainsi l'élément explicatif de l'état de souffrance au travail vécue par ces jeunes, une 
temporalité perçue comme transitoire ou du futur proximal. Si la rupture est produite par la souffrance, les auteurs se demandent justement s'il n'est pas plus raisonné d'interpréter cette rupture comme une expérience positive, comme une échappatoire ou un moment libérateur qui autorise ces jeunes à réévaluer leurs options d'insertion professionnelle.

Josette Kodsi et Marc Molgat se questionnent sur la place de l'école et du travail dans la quête identitaire des adolescentes et des adolescents d'aujourd'hui. La construction identitaire des jeunes est marquée à notre époque par le passage d'un déterminisme institutionnel à une plus grande liberté individuelle, accompagné par la perte de repères et l'apparition de nombreuses incertitudes. Malgré cette individualisation des parcours biographiques, les individus, et implicitement les adolescents, se retrouvent toujours dans la situation de s'adapter à des normes sociales et de subir les pressions sociales. Les incertitudes spécifiques pour notre société sont alors amplifiées par l'éclosion de nouvelles pressions, comme la norme de prolongement des études ou la poussée pour rejoindre le marché du travail ou encore l'incitation à manifester son autonomie par la consommation. Ces réflexions ont façonné la lecture des résultats d'une enquête exploratoire effectuée auprès des élèves des classes terminales d'une école secondaire francophone de la région d'Ottawa. Si l'école est vue par ces jeunes presque exclusivement dans une perspective utilitaire, comme une étape incontournable du processus d'insertion professionnelle, le travail prend une signification beaucoup plus riche et valorisée : c'est une source de satisfaction, d'expression et d'autonomie, une occasion pour manifester son sens des responsabilités et un moyen d'accumuler des expériences professionnelles. Ces résultats illustrent la façon dont les adolescentes et les adolescents "s'adaptent" aux normes d'insertion professionnelle afin de générer des "certitudes biographiques ». Pour mener à bien leur stabilisation identitaire, les jeunes érigent ce travail symbolique sur un fondement normatif offert par un environnement social éclaté. Comme résultat, l'investissement dans le travail et les études se fait au détriment des loisirs et des relations sociales, pourtant nécessaires dans un contexte social marqué par l'incertitude. 
À la fin des études secondaires, les jeunes prennent déjà des décisions très importantes pour favoriser leur insertion socioprofessionnelle. Décider d'entrer sur le marché du travail, de continuer des études postsecondaires ou de choisir le domaine de spécialisation implique déjà l'existence d'une représentation assez bien définie de la direction professionnelle à envisager. L'étude de Lilian Negura et d'André Samson sur la représentation sociale du travail des jeunes francophones de l'Ontario trouve son fondement dans l'importance de ce contenu représentationnel pour la qualité des décisions prises par les jeunes à cette étape de leur vie. L'enquête par entrevue semi-dirigée auprès de treize élèves provenant de quatre écoles secondaires francophones de différentes régions de l'Ontario a permis l'identification de trois aspects de la représentation sociale du travail. L'aspect descriptif a mis en relief une vision assez pessimiste du marché du travail, comme réalité précaire et incertaine, qui, malgré tout, reflète le discours scientifique en la matière. L'aspect normatif souligne l'importance pour ces jeunes de la réalisation personnelle par le travail, plus significative que la simple obtention d'un revenu. Cette tendance qui prédomine l'imaginaire de la génération Y, pourrait avoir des conséquences imprévisibles sur la configuration du travail de demain. Et, finalement, l'appartenance à la francophonie fait ressortir des conséquences inattendues de l'aspect identitaire de la représentation sociale du travail des jeunes. Dans le contexte minoritaire ontarien, le bilinguisme des francophones est vu comme une distinction structurelle positive qui facilite l'intégration au marché du travail. Cependant, selon les conclusions des auteurs, le bilinguisme ne fait que renforcer l'identité francophone de ces jeunes Ontariens.

Rachid Merzouk ajoute une autre dimension aux problématiques soulevées par ce dossier en présentant une analyse de l'exclusion sociale et comment Catherine, victime d'un handicap à la naissance, doit lutter de façon héroïque pour se tailler une place dans une société axée sur la performance, la rapidité, la perfection. En se basant sur le témoignage de cette jeune adulte, l'auteur identifie les difficultés et défis rattachés à chaque étape de son évolution vers le monde du travail : sa tendre enfance et les 
résistances de ses parents à ce qu'elle accepte son handicap, l'école et ses contradictions structurelles d'apprentissage, l'université et les défis d'une formation axée sur la performance et enfin, la recherche d'un emploi dans le contexte où les employeurs démontrent clairement leurs réticences : voulant une personne performante, ils se centrent sur son handicap bien plus que sur son potentiel. Le texte démontre en quel sens le rêve de Catherine de devenir enseignante est constamment mis à l'épreuve; son courage finit par flancher, en quelque sorte, face aux murs de résistance de ses "malheureuses expériences professionnelles". Il devient évident que le monde du travail ne semble pas prêt à accepter une éducatrice possédant un handicap visible qui ralentirait peut-être son rythme de fonctionnement, mais qui en même temps démontrerait aux jeunes une ouverture à l'autre. Rachid Merzouk privilégie une analyse de sociologie clinique de l'exclusion sociale pour nous aider à décoder les diverses étapes que doit franchir toute personne confrontée à un handicap visible, défi qui s'ajoute aux difficultés habituelles d'insertion professionnelle. Son texte démontre comment « les expériences de discrimination et de rejet vécues en lien avec le travail agissent sur la personne en résonnance avec d'autres expériences de même nature précédemment vécues ", ensemble de facteurs qu'il qualifie de "métasituation", ce qui fait en sorte que Catherine, comme bien d'autres, reste prisonnière des contradictions sociales qui s'abattent sur elles.

Dans cet article comme dans les précédents, l'intervention sociale prend une place de choix dans le sens où chaque auteur et auteure propose des pistes de renouvellement des pratiques d'intervention qui répondent aux problèmes présentés.

\section{Les pratiques à notre image}

Dans ce dossier, trois personnes d'expérience partagent leurs réflexions face aux réalités changeantes du marché de l'emploi en service social. 
Joscelyne Levesque décrit le cheminement qui l'a amenée à adopter un regard critique face à un marché du travail qui privilégie l'uniformité et la conformité aux normes institutionnelles plutôt que l'originalité et la réflexion critique. La recherche de sens qu'elle a entreprise au début de sa carrière l'amène à poser la question à savoir si son travail, en service social, est libérateur ou endroit d'oppression. Sa démarche franche et courageuse débouche sur des réflexions qui en fait résument l'impasse de plusieurs intervenants-chercheurs : comment travailler le social en service social? Elle propose la poursuite individuelle et collective d'un rêve visant à "construire une société où un projet collectif réunissant les valeurs de justice sociale, de solidarité et d'inclusion sociale au service du bien commun ».

Lorraine Brissette pour sa part propose une réflexion sur l'épuisement professionnel dans le contexte de contraintes structurelles de plus en plus serrées en service social. Son article présente les réflexions d'une gestionnaire et auteure qui se spécialise depuis une décennie sur la question de l'épuisement des intervenantes et intervenants dans le domaine des services de santé et des services sociaux. Les publications de l'auteure sur ce sujet datent de la fin des années 90 . Dix ans après, qu'en est-il de la situation dans un contexte social, politique et culturel qui a beaucoup évolué? Quelles ont été les conséquences des changements qu'elle mentionne sur les risques associés à l'épuisement des travailleurs sociaux? «Les jeunes travailleurs ne vivent plus pour travailler, mais travaillent pour vivre. Cela ne signifie pas qu'ils ne sont pas impliqués dans leur travail, mais ils revendiquent un meilleur équilibre entre leur vie personnelle et professionnelle "; voilà une des constatations qui émanent de son texte. Forte de son expérience d'intervenante et de consultante, Lorraine Brissette propose des balises permettant aux travailleurs et travailleuses de prévenir l'épuisement professionnel tout en restant engagés dans leur profession.

Les réflexions de Stéphane Richard au sujet de la délibération éthique en contexte d'intervention difficile démontrent que "l'éthique n'est pas quelque chose qu'on joue, mais ce que l'on incarne». L'auteur démontre que, confrontés à des situations 
d'intervention critiques où ils et elles doivent faire des choix qui mettent à l'épreuve leurs compétences décisionnelles et leur souci de responsabilité, les professionnelles et professionnels ont recours à des repères personnels, normatifs, administratifs, mais aussi éthiques. En s'inspirant de plusieurs textes classiques sur le sujet, Richard démontre que le fait d'être disposé à s'exposer à la délibération éthique suscite des actes éthiques spontanés, considérés comme l'expression d'un savoir-faire, manifestation d'attitudes éthiques agissantes.

\section{En guise de conclusion}

Après avoir lu ce numéro, nous espérons que le lecteur sera en mesure de mieux saisir certaines des dimensions d'un monde du travail en constante évolution. Les différentes questions que posent ces articles, à toutes sortes de niveaux, nous permettent de conclure sur l'urgence d'une réflexion d'ensemble sur un sujet qui, il va sans dire, soulève des débats importants pour notre profession, mais aussi pour le renouvellement d'un monde du travail qui n'a sans doute pas toujours évalué la complexité des problématiques sociales qu'il soulève.

\section{Bibliographie}

CASTEL, Robert (2000). Les métamorphoses de la question sociale. Une chronique du salariat, Paris, Folio-Gallimard.

CHAUVEL, Louis (2006). Les classes moyennes à la dérive, Paris, Seuil.

DEJOURS, Christophe (2003). L'évaluation du travail à l'épreuve du réel. Critique des fondements de l'évaluation Dijon, INRA Éditions.

DUBAR, Claude (2000). La crise des identités. L'interprétation d'une mutation. Paris, Presses Universitaires de France.

DUVAL, Guillaume, et Henri JACOT (2000). Le travail dans la société de l'information. Paradoxes et enjeux des Nouvelles Technologies d'Information et de Communication, Paris, Éditions Liaison.

FOURNIER, Geneviève, Bruno BOURASSA et Kamel BÉJI (2003). «Travail atypique récurrent et expérience de précarité : un regard exploratoire " dans Fournier, Bourassa et Béji, La précarité du travail. Une réalité aux multiples visages, Québec, Les Presses de l’Université Laval. 
GRENIER, André (1998). Les jeunes et le marché du travail : tendance et situation récente, Québec, Direction de l'analyse du marché du travail et de l'évaluation, Emploi-Québec.

HANGO, Darcy, et Patrice de BROUCKER (2007). Cheminements des jeunes Canadiens des études au marché du travail : résultats de l'enquête auprès des jeunes en transition, Ottawa, Statistique Canada.

GUIBERT, Philippe, et Alain MER GIER (2006). Le descenseur social. Enquête sur les milieux populaires, Paris, FJJ/Plon.

LECOMTE, Roland (2000). "L'évolution du travail social : une histoire à suivre ", Reflets, Revue ontaroise d'intervention sociale et communautaire,Vol. 6, No 1, p. 18-34.

MOLINIER, Pascale (2007). Les enjeux psychiques du travail : introduction à la psychodynamique du travail, Paris, Payot.

MOULIER-BOUTANG, Yann (2007). Le capitalisme cognitif. La nouvelle grande transformation, Paris, Editions Amsterdam.

NEGURA, Lilian (2006). "L'évolution de la représentation sociale du travail dans le contexte de mutations économiques en Occident ", Carrièrologie, 10(3), p. 393-410.

PAUGAM, Serge (2002). La disqualification sociale. Essai sur la nouvelle pauvreté, Paris, Presses Universitaires de France.

PERNOT, Jean-Marie (2005). Syndicats : lendemains de crise?, Paris, Gallimard.

RAYMOND, Mélanie (2008). Décrocheurs du secondaire retournant à l'école, Ottawa, Statistique Canada, No 81-595-MIF2008055 au catalogue.

RINEHART, James W. (2001). The Tyranny of WORK. Alienations and the Labour Process, Toronto, Montréal, Harcourt Canada.

ROSANVALLON, Pierre (1995). La nouvelle question sociale, Paris, Le Seuil.

ROSE, José (2000). Disparition ou transformation des formes de l'emploi, Sainte-Foy, Les Presses de l'Université Laval.

STATISTIQUE CANADA (2004). Information sur la population active : Décembre 2003, Statistique Canada, No 71-001-XIF au catalogue.

TREMBLAY, Diane-Gabrielle, et collab. (2003). «Les politiques d'emploi à l'intention des jeunes au Canada et en Australie : plus ou moins de vulnérabilité et de précarité? " dans Fournier, Bourassa et Béji, La précarité du travail. Une réalité aux multiples visages, Québec, Les Presses de l'Université Laval.

\section{Notes}

1. Une expression consacrée dans les sciences sociales francophones pour désigner la période qui s'échelonne entre 1950 et 1980 environ.

2. Le taux de chômage des décrocheurs du secondaire (pour le groupe des 15 ans et plus) a été d'environ $17 \%$ en 2004. Source : tableau CANSIM 282-0004, Statistique Canada. 